\title{
DYNAMICAL WEYL GROUPS AND EQUIVARIANT COHOMOLOGY OF TRANSVERSAL SLICES ON AFFINE GRASSMANNIANS
}

\author{
Alexander Braverman and Michael Finkelberg
}

\begin{abstract}
Let $G$ be a reductive group and let $\breve{G}$ be its Langlands dual. We give an interpretation of the dynamical Weyl group of $\breve{G}$ defined in [5] in terms of the geometry of the affine Grassmannian Gr of $G$. In this interpretation the dynamical parameters of [5] correspond to equivariant parameters with respect to certain natural torus acting on Gr. We also present a conjectural generalization of our results to the case of affine Kac-Moody groups.
\end{abstract}

\section{Introduction and statements of the results}

1.1. Notations and overview. We mainly follow the notations of [3]. So $G \supset$ $B \supset T$ is a reductive complex algebraic group with a Borel subgroup, and a Cartan subgroup. Let $\breve{G} \supset \check{T}$ denote the Langlands dual group with its Cartan torus. Let $\mathcal{K}=\mathbb{C}((t)), \quad \mathcal{O}=\mathbb{C}[[t]]$. The affine Grassmannian $\mathrm{Gr}=\mathrm{Gr}_{G}=G(\mathcal{K}) / G(\mathcal{O})$ carries the category $\operatorname{Perv}_{G(\mathcal{O})}(\mathrm{Gr})$ of $G(\mathcal{O})$-equivariant perverse constructible sheaves. It is equipped with the convolution monoidal structure, and is tensor equivalent to the tensor category $\operatorname{Rep}(\check{G})$ of representations of $\check{G}$ (see [8], [6], [10], [2]). We denote by $\tilde{S}: \operatorname{Rep}(\check{G}) \rightarrow \operatorname{Perv}_{G(\mathcal{O})}(\mathrm{Gr})$ the geometric Satake isomorphism functor, and by $S: \operatorname{Rep}(\breve{G}) \rightarrow \operatorname{Perv}_{G(\mathcal{O}) \rtimes \mathbb{C}^{*}}(\mathrm{Gr})$ its extension to the monoidal category of $G(\mathcal{O}) \rtimes \mathbb{C}^{*}-$ equivariant perverse constructible sheaves.

The Lie algebras of $\check{G} \supset \check{T}$ are denoted by $\check{\mathfrak{g}} \supset \check{\mathfrak{t}}$. We have $\check{\mathfrak{t}}=\mathfrak{t}^{*}$ canonically. The Weyl group of $G, \breve{G}$ is denoted by $W$. Let $\Lambda=\Lambda_{G}$ denote the coweight lattice of $T$ which is the same as the weight lattice of $\check{T}$. The choice of Borel subgroup $B \subset G$ defines the cone $\Lambda_{G}^{+} \subset \Lambda_{G}$ of dominant weights of $\check{T}$.

The lattice $\Lambda=\Lambda_{G}$ is identified with the quotient $T(\mathcal{K}) / T(\mathcal{O})$. For $\lambda \in \Lambda$ we denote by $t^{\lambda}$ any lift of $\lambda$ to $T(\mathcal{K})$. Its image in $\operatorname{Gr}_{G}=G(\mathcal{K}) / G(\mathcal{O})$ is independent of the choice of a lift, and will be also denoted by $t^{\lambda}$, or sometimes just $\lambda \in \operatorname{Gr}_{G}$. Moreover, we will keep the same name for the closed embedding $\lambda \hookrightarrow$ Gr. Let $\operatorname{Gr}^{\lambda}$ denote the $G(\mathcal{O})$-orbit of $\lambda$, and let $\overline{\mathrm{Gr}}^{\lambda} \subset \mathrm{Gr}$ denote the closure of $\mathrm{Gr}^{\lambda}$. It is well known that $\mathrm{Gr}=\bigcup_{\lambda \in \Lambda} \mathrm{Gr}^{\lambda}$, and that $\mathrm{Gr}^{\lambda}=\mathrm{Gr}^{\mu}$ iff $\lambda$ and $\mu$ lie in the same $W$-orbit on $\Lambda$. In particular, $\mathrm{Gr}=\bigsqcup_{\lambda \in \Lambda^{+}} \mathrm{Gr}^{\lambda}$.

It is known that as a byproduct of the existence of $S$ (or $\widetilde{S}$ ) one can get a geometric construction of finite-dimensional representations of $\breve{G}$. It is not difficult to note

Received by the editors February 16, 2011. 
that this realization immediately gives rise to some additional structures on finitedimensional representations of $\breve{G}$ and one may wonder what is the representationtheoretic meaning of those finer structures. In this paper we are going to explore one such example. Namely, let $V \in \operatorname{Rep}(\check{G})$ and let $\mu$ be a weight of $V$, i.e. an element of $\Lambda$ such that $V_{\mu} \neq 0$. Let also $w \in W$. Then we explain in Section 1.3 that from the geometry of Gr one gets a canonical isomorphism $V_{\mu} \otimes \mathbb{C}\left(\mathfrak{t} \times \mathbb{A}^{1}\right) \rightarrow V_{w \mu} \otimes \mathbb{C}\left(\mathfrak{t} \times \mathbb{A}^{1}\right)$. Here $\mathbb{A}^{1}$ is the affine line and $\mathbb{C}\left(\mathfrak{t} \times \mathbb{A}^{1}\right)$ denotes the field of rational functions on $\mathfrak{t} \times \mathbb{A}^{1}$. The main purpose of this paper is to show that when $\mu \in \Lambda^{+}$the above isomorphism coincides (up to some simple reparametrization) with the one provided by the dynamical Weyl group constructed in [5].

1.2. More notation. For $\lambda, \mu \in \Lambda^{+}$, we have $\overline{\mathrm{Gr}}^{\mu} \subset \overline{\mathrm{Gr}}^{\lambda}$ iff $\mu \leq \lambda$, i.e. $\lambda-\mu$ is a sum of positive roots of $\check{G}$. We denote by $G\left[t^{-1}\right]_{1} \subset G\left[t^{-1}\right] \subset G(\mathcal{K})$ the kernel of the natural ("evaluation at $\infty$ ") homomorphism $G\left[t^{-1}\right] \rightarrow G$. For $\mu \in \Lambda$ we set $\operatorname{Gr}_{\mu}=G\left[t^{-1}\right] \cdot \mu \subset \mathrm{Gr}$, and $\mathcal{W}_{\mu}=G\left[t^{-1}\right]_{1} \cdot \mu$. The locally closed embedding $\mathcal{W}_{\mu} \hookrightarrow$ Gr will be denoted by $\mathfrak{i}_{\mu}$. For dominant $\mu \leq \lambda \in \Lambda^{+}$, we set $\mathcal{W}_{\mu}^{\lambda}=\operatorname{Gr}^{\lambda} \cap \mathcal{W}_{\mu}$, and $\overline{\mathcal{W}}_{\mu}^{\lambda}=\overline{\mathrm{Gr}}^{\lambda} \cap \mathcal{W}_{\mu}$. The variety $\overline{\mathcal{W}}_{\mu}^{\lambda}$ can be thought of as a transversal slice to $\mathrm{Gr}^{\mu}$ inside $\overline{\mathrm{Gr}^{\lambda}}$.

For $V \in \operatorname{Rep}(\check{G})$ we can restrict the $G(\mathcal{O}) \rtimes \mathbb{C}^{*}$-equivariant perverse sheaf $S(V)$ to the transversal slice $\mathcal{W}_{\mu}$. We have $\mathfrak{i}_{\mu}^{*} S(V)\left[-\operatorname{dim} \mathrm{Gr}^{\mu}\right] \simeq \mathfrak{i}_{\mu}^{!} S(V)\left[\operatorname{dim} \mathrm{Gr}^{\mu}\right]$, and we will denote this restriction by $S_{\mu}(V)$. It is a $T \times \mathbb{C}^{*}$-equivariant perverse sheaf on $\mathcal{W}_{\mu}$.

Let $T \subset B_{-} \subset G$ be the opposite (to $T \subset B \subset G$ ) Borel subgroup, and let $N_{-} \subset B_{-}$be its unipotent radical. For $w \in W$ we consider the conjugate subgroup $N_{-}^{w}:=\tilde{w}^{-1} N_{-} \tilde{w}$ for any lift $\tilde{w}$ of $w$ to the normalizer of $T$. For $\lambda \in \Lambda$ we set $\mathfrak{T}_{\lambda}^{w}=N_{-}^{w}(\mathcal{K}) \cdot \lambda \subset$ Gr. The locally closed embedding $\mathfrak{T}_{\lambda}^{w} \hookrightarrow$ Gr is denoted by $\imath_{\lambda}^{w}$, and the closed embedding $\lambda \hookrightarrow \mathfrak{T}_{\lambda}^{w}$ is denoted by $\mathfrak{j}_{\lambda}^{w}$.

For a $T \times \mathbb{C}^{*}$-equivariant constructible complex $\mathcal{F}$ on $\mathrm{Gr}$, we denote by $R_{\mu}^{w} \mathcal{F}$ the $H_{T \times \mathbb{C}^{*}}^{\bullet}(p t)$-module $\mathfrak{j}_{\mu}^{w *} \imath_{\mu}^{w !} \mathcal{F}[\langle w \mu, 2 \check{\rho}\rangle]$. Here $2 \check{\rho}$ is the sum of positive roots of $G$, and $H_{T \times \mathbb{C}^{*}}^{\bullet}(p t) \simeq \mathbb{C}\left[\mathfrak{t} \times \mathbb{A}^{1}\right]$. We denote by $r_{\mu}^{w} \mathcal{F}$ the vector space $\mathfrak{j}_{\mu}^{w *} \imath_{\mu}^{w !} \mathcal{F}[\langle w \mu, 2 \check{\rho}\rangle]$ with $T \times \mathbb{C}^{*}$-equivariance forgotten. Thus, $r_{\mu}^{w} \mathcal{F}$ is the fiber of $R_{\mu}^{w} \mathcal{F}$ over $0 \in \mathfrak{t} \times \mathbb{A}^{1}$. Similarly, for a $T \times \mathbb{C}^{*}$-equivariant constructible complex $\mathcal{F}$ on $\mathcal{W}_{\mu}$, we denote by $\mathrm{R}_{\mu}^{w} \mathcal{F}$ the $H_{T \times \mathbb{C}^{*}}^{\bullet}(p t)$-module $\mathfrak{j}_{\mu}^{w *} \imath_{\mu}^{w !} \mathcal{F}$. We denote by $\mathrm{r}_{\mu}^{w} \mathcal{F}$ the vector space $\mathfrak{j}_{\mu}^{w *} \imath_{\mu}^{w !} \mathcal{F}$ with $T \times \mathbb{C}^{*}$-equivariance forgotten. Thus, $r_{\mu}^{w} \mathcal{F}$ is the fiber of $\mathrm{R}_{\mu}^{w} \mathcal{F}$ over $0 \in \mathfrak{t} \times \mathbb{A}^{1}$.

1.3. Hyperbolic stalks. Recall that for $V \in \operatorname{Rep}(\check{G})$ we have a $T \times \mathbb{C}^{*}$-equivariant perverse sheaf $S_{\mu}(V)$ on the slice $\mathcal{W}_{\mu}$. The equivariant costalk $\mu^{!} S_{\mu}(V)=\mathfrak{j}_{\mu}^{w !} \imath_{\mu}^{w !} S_{\mu}(V)$ is an $H_{T \times \mathbb{C}^{*}}^{\bullet}(p t)$-module equipped with a natural morphism $C_{\mu}^{w}$ to $\mathfrak{j}_{\mu}^{w *} \imath_{\mu}^{w !} S_{\mu}(V)=$ $\mathrm{R}_{\mu}^{w} S_{\mu}(V)$. Note that $C_{\mu}^{w}$ is a morphism of $H_{T \times \mathbb{C}^{*}}^{\bullet}(p t)=\mathbb{C}\left[\mathfrak{t} \times \mathbb{A}^{1}\right]$-modules. If we extend the scalars to the field of fractions $\mathbb{C}\left(\mathfrak{t} \times \mathbb{A}^{1}\right)$, the morphism $C_{\mu}^{w}$ becomes an isomorphism due to Localization Theorem in equivariant cohomology (note that $\mu$ is the unique $T$-fixed point of $\left.\mathcal{W}_{\mu}\right)$. For $w, y \in W$, we denote by $\mathrm{A}_{w, y}^{S_{\mu}(V)}$ : $\mathrm{R}_{\mu}^{w} S_{\mu}(V) \otimes_{\mathbb{C}\left[\mathfrak{t} \times \mathbb{A}^{1}\right]} \mathbb{C}\left(\mathfrak{t} \times \mathbb{A}^{1}\right) \stackrel{\sim}{\longrightarrow} \mathrm{R}_{\mu}^{y} S_{\mu}(V) \otimes_{\mathbb{C}\left[\mathfrak{t} \times \mathbb{A}^{1}\right]} \mathbb{C}\left(\mathfrak{t} \times \mathbb{A}^{1}\right)$ the composition $C_{\mu}^{y} \circ\left(C_{\mu}^{w}\right)^{-1}$.

Lemma 1.4. (a) For $w \in W, \mu \in \Lambda, V \in \operatorname{Rep}(\check{G})$, we have a canonical isomorphism $\mathrm{r}_{\mu}^{w} S_{\mu}(V) \cong V_{w \mu}$ (the $w \mu$-weight space of $V$ ); 
(b) For $w \in W, \mu \in \Lambda, V \in \operatorname{Rep}(\check{G})$, we have a canonical isomorphism $\mathrm{R}_{\mu}^{w} S_{\mu}(V) \cong$ $\mathrm{r}_{\mu}^{w} S_{\mu}(V) \otimes \mathbb{C}\left[\mathfrak{t} \times \mathbb{A}^{1}\right]$.

According to Lemma 1.4, we can view the isomorphism $A_{w, y}^{S_{\mu}(V)}$ as going from $V_{w \mu} \otimes \mathbb{C}\left(\mathfrak{t} \times \mathbb{A}^{1}\right)$ to $V_{y \mu} \otimes \mathbb{C}\left(\mathfrak{t} \times \mathbb{A}^{1}\right)$. Let us denote by $\hbar \in \mathbb{C}\left[\mathbb{A}^{1}\right]$ the positive generator of $H_{\mathbb{C}^{*}}^{2}(p t, \mathbb{Z})$.

1.5. Dynamical Weyl group. The dynamical Weyl group operators for simple Lie algebras were introduced in [12] by analogy with extremal projectors of [1]. We will follow the presentation of [5]. We refer the reader to loc. cit. for the discussion of numerous applications of the dynamical Weyl group. Let $U_{\hbar}$ be the "graded enveloping" algebra of $\check{\mathfrak{g}}$, i.e. the graded $\mathbb{C}[\hbar]$-algebra generated by $\check{\mathfrak{g}}$ with relations $x y-y x=\hbar[x, y]$ for $x, y \in \check{\mathfrak{g}}$. It is a subalgebra of $U(\mathfrak{\mathfrak { g }}) \otimes \mathbb{C}[\hbar]$. Any $V \in \operatorname{Rep}(\breve{G})$ gives rise to a structure of $U_{\hbar}$-module on $V \otimes \mathbb{C}[\hbar]$, and then the construction of [5] (Section 4, Main Definition 2) produces the dynamical Weyl group operators $A_{w, V}: V \otimes \mathbb{C}\left(\check{\mathfrak{t}}^{*} \times \mathbb{A}^{1}\right) \rightarrow V \otimes \mathbb{C}\left(\check{\mathfrak{t}}^{*} \times \mathbb{A}^{1}\right)$. Here $\mathbb{A}^{1}$ is the affine line with coordinate $\hbar$. Note that for $\mu \in \Lambda$, the operator $A_{w, V}$ takes the weight space $V_{\mu} \otimes \mathbb{C}\left(\check{\mathfrak{t}}^{*} \times \mathbb{A}^{1}\right)$ to the weight space $V_{w \mu} \otimes \mathbb{C}\left(\check{\mathfrak{t}}^{*} \times \mathbb{A}^{1}\right)$.

Recall that $\check{\mathfrak{t}}^{*}=\mathfrak{t}$, and let $2 \rho \in \check{\mathfrak{t}}^{*}=\mathfrak{t}$ be the sum of all positive roots of $\breve{G}$. Let $e$ stand for the neutral element of the Weyl group $W$. The following is the main result of this paper:

Theorem 1.6. Let $V \in \operatorname{Rep}(\breve{G}), \mu \in \Lambda^{+}$. For $x \in \mathfrak{t} \times \mathbb{A}^{1}$ we have $\mathrm{A}_{e, w}^{S_{\mu}(V)}(x)=$ $A_{w, V}(-x-\hbar \rho)$. This is an equality of rational functions on $\mathfrak{t} \times \mathbb{A}^{1}$ with values in $\operatorname{Hom}\left(V_{\mu}, V_{w \mu}\right)$.

1.7. Organization of the paper. Section 2 is devoted to the proof of Theorem 1.6. Let us note that the only proof we have at the moment is by means of some "brute force" calculation. In particular, we don't use the elegant definition of $A_{w, V}$ via intertwining operators between certain Verma-type modules over $\check{\mathfrak{g}}$ given in [5]; rather by using some formal properties of both $A_{w, V}$ and $\mathrm{A}_{w, y}^{S_{\mu}(V)}$ we reduce Theorem 1.6 to the case of $G=P G L(2)$ where the two sides are compared by means of an explicit calculation (for $A_{w, V}$ this calculation is done in [5]). It would be interesting to find a more conceptual proof of Theorem 1.6.

In Section 3 we formulate a conjectural generalization of Theorem 1.6 to the case of affine Kac-Moody groups (based on the construction of affine analogs of $\overline{\mathcal{W}}_{\mu}^{\lambda}$ given in $[4])$.

1.8. Acknowledgments. The idea of this work was born after a series of conversations we had with D. Maulik and A. Okounkov, who explained to us the contents of their work in progress [9]; in particular, one of the (numerous) results of [9] probably implies a proof of our Conjecture 3.3 in the case of level 1 representations for $G=G L(n)$. In fact, one of the main purposes of this paper was to put this result in some more general framework. We would like to thank D. Maulik and A. Okounkov for their patient explanations of the contents of [9] to us.

We are also grateful to P. Etingof for teaching us about the dynamical Weyl group. We are happy to thank the IAS at the Hebrew University of Jerusalem for the excellent working conditions. M. F. was partially supported by the RFBR grant 09-01-00242, 
the Ministry of Education and Science of Russian Federation, grant No. 2010-1.3.1111-017-029, and the AG Laboratory HSE, RF government grant, ag. 11.G34.31.0023.

\section{Proofs}

2.1. Proof of Lemma 1.4. (a) By definition of the Mirković-Vilonen fiber functor, we have $r_{\mu}^{e} S(V)=V_{\mu}$. Due to the $G(\mathcal{O})$-equivariance of the sheaf $S(V)$, we have $r_{\mu}^{w} S(V) \cong r_{w \mu}^{e} S(V)=V_{w \mu}$. Let $\lambda \in \Lambda^{+}$be big enough so that $\overline{\mathrm{Gr}^{\lambda}}$ contains the support of $S(V)$. According to Propositions 1.3.1 and 1.3.2 of [7], there is a $T \times$ $\mathbb{C}^{*}$-invariant open subset $U^{\mu} \subset \overline{\mathrm{Gr}^{\mu}}$ containing $\mu$ and an open $T \times \mathbb{C}^{*}$-equivariant embedding $U_{\mu}^{\lambda}:=U^{\mu} \times \overline{\mathcal{W}}_{\mu}^{\lambda} \hookrightarrow \overline{\mathrm{Gr}}^{\lambda}$.

Recall that $2 \rho$ stands for the sum of all positive roots of $\check{G}$. We view $2 \rho$ as a cocharacter $\mathbb{C}^{*} \rightarrow T$. Then $\mathfrak{T}_{\mu}^{w}$ is the repellent of the point $\mu \in \mathrm{Gr}$ with respect to the one-parametric subgroup $2 w \rho: \mathbb{C}^{*} \rightarrow T$, i.e. $\mathfrak{T}_{\mu}^{w}=\left\{g \in \mathrm{Gr}: \lim _{z \rightarrow \infty} 2 w \rho(z) g=\mu\right\}$ (see [10], (3.5)). It follows that $\mathfrak{T}_{\mu}^{w} \cap U_{\mu}^{\lambda}=\left(\mathfrak{T}_{\mu}^{w} \cap U^{\mu}\right) \times\left(\mathfrak{T}_{\mu}^{w} \cap \overline{\mathcal{W}}_{\mu}^{\lambda}\right)$. Now $\left.S(V)\right|_{U_{\mu}^{\lambda}} \cong$ $\left.\mathrm{IC}\left(\overline{\mathrm{Gr}}^{\mu}\right)\right|_{U^{\mu}} \otimes S_{\mu}(V)$. We conclude that $r_{\mu}^{w} S(V) \cong r_{\mu}^{w} \mathrm{IC}\left(\overline{\mathrm{Gr}}^{\mu}\right) \otimes \mathrm{r}_{\mu}^{w} S_{\mu}(V)$.

Now $\mathfrak{T}_{\mu}^{w} \cap \overline{\mathrm{Gr}}^{\mu}=N_{-}^{w}(\mathcal{O}) \cdot \mu$ (see [10], proof of Theorem 3.2) is an affine space contained in $\mathrm{Gr}^{\mu}$. So $\mathrm{IC}\left(\overline{\mathrm{Gr}}^{\mu}\right)$ is constant along $\mathfrak{T}_{\mu}^{w} \cap \overline{\mathrm{Gr}}^{\mu}$, and we have canonically $r_{\mu}^{w} \mathrm{IC}\left(\overline{\mathrm{Gr}}^{\mu}\right) \cong \mathbb{C}$. Thus, $V_{w \mu} \cong r_{\mu}^{w} S(V) \cong \mathbb{C} \otimes \mathrm{r}_{\mu}^{w} S_{\mu}(V)=\mathrm{r}_{\mu}^{w} S_{\mu}(V)$. The proof of (a) is complete.

To prove (b) note that by (a) and Theorem 3.5 of $[10], r_{\mu}^{w} S_{\mu}(V)$ is concentrated in degree 0 and coincides with the degree zero part of $\mathrm{R}_{\mu}^{w} S_{\mu}(V)$. Thus the natural map (the action) $\mathrm{r}_{\mu}^{w} S_{\mu}(V) \otimes H_{T \times \mathbb{C}^{*}}^{\bullet}(p t) \rightarrow \mathrm{R}_{\mu}^{w} S_{\mu}(V)$ is the desired isomorphism. The proof of the lemma is complete.

2.2. Proof of Theorem $\mathbf{1 . 6}$ for $G=P G L(2)$. For $G=P G L(2)$ we have $\check{G}=$ $S L(2)$, and let $V=V^{\lambda}$ be the irreducible representation of $\breve{G}$ with highest weight $\lambda \in \mathbb{N}$, and highest vector $v_{\lambda}$. We consider the basis $\left\{v_{\mu}, \mu=-\lambda,-\lambda+2, \ldots, \lambda-2, \lambda\right\}$ of $V^{\lambda}$ such that $v_{\lambda-2 k}=\frac{f^{k}}{k !} v_{\lambda}$ where $(e, h, f)$ is the standard basis of $\mathfrak{s l}(2)$. In this basis, the action of dynamical Weyl group is computed in Propositions 6 and 12 of [5]. If $s$ is the nontrivial element of $W=\mathbb{Z} / 2 \mathbb{Z}$, and $\mu \geq 0$, then

$$
A_{s, V^{\lambda}}(x) v_{\mu}=(-1)^{\frac{\lambda-\mu}{2}} \frac{(x+2 \hbar)(x+3 \hbar) \ldots\left(x+\hbar+\frac{\lambda-\mu}{2} \hbar\right)}{\left(x+\hbar-\frac{\lambda+\mu}{2} \hbar\right)\left(x+2 \hbar-\frac{\lambda+\mu}{2} \hbar\right) \ldots(x-\mu \hbar)} v_{-\mu}
$$

Here $x \in \mathbb{C}[\mathfrak{t}]$ stands for the linear function $\langle\cdot, \check{\alpha}\rangle$ where $\check{\alpha}$ is the positive simple root of $G$. We see that

$$
A_{s, V^{\lambda}}(-x-\hbar) v_{\mu}=\frac{(x-\hbar)(x-2 \hbar) \ldots\left(x-\frac{\lambda-\mu}{2} \hbar\right)}{\left(-x-\frac{\lambda+\mu}{2} \hbar\right)\left(-x+\hbar-\frac{\lambda+\mu}{2} \hbar\right) \ldots(-x-\hbar-\mu \hbar)} v_{-\mu}
$$

We have $S\left(V^{\lambda}\right)=\mathbb{C}_{\overline{\overline{G r}^{\lambda}}}[\lambda]$, and $V^{\lambda}=H^{\bullet}(\overline{\mathrm{Gr}} \lambda, \mathbb{C}[\lambda])$. The basis $\left\{v_{\mu}\right\}$ consists of the (Poincaré duals) of the fundamental classes of the closures of intersections $\mathfrak{T}_{\mu}^{e} \cap \overline{\mathrm{Gr}^{\lambda}}$ (see e.g. Section 5.2 of [3]). We conclude from the proof of Lemma 1.4 that under the isomorphism $\mathrm{R}_{\mu}^{e} S_{\mu}\left(V^{\lambda}\right) \cong V_{\mu}^{\lambda} \otimes \mathbb{C}[x, \hbar]\left(\right.$ resp. $\left.\mathrm{R}_{\mu}^{s} S_{\mu}\left(V^{\lambda}\right) \cong V_{-\mu}^{\lambda} \otimes \mathbb{C}[x, \hbar]\right)$ the positive generator of the cohomology with integral coefficients goes to $v_{\mu}$ (resp. to $v_{-\mu}$ ). Here $x$ stands for the positive generator of the integral equivariant cohomology $H_{T}^{2}(p t, \mathbb{Z})$. Let $1_{\mu}$ stand for the unit element in the equivariant costalk $\mu^{!} \underline{\mathbb{C}}_{\overline{\mathrm{Gr}^{\lambda}}}[\lambda]$. 
Then

$$
C_{\mu}^{e}\left(1_{\mu}\right)=\left(-x-\frac{\lambda+\mu}{2} \hbar\right)\left(-x+\hbar-\frac{\lambda+\mu}{2} \hbar\right) \ldots(-x-\hbar-\mu \hbar) v_{\mu},
$$

while

$$
C_{\mu}^{s}\left(1_{\mu}\right)=(x-\hbar)(x-2 \hbar) \ldots\left(x-\frac{\lambda-\mu}{2} \hbar\right) v_{-\mu} .
$$

In effect, $\mathfrak{T}_{\mu}^{e} \cap \overline{\mathcal{W}}_{\mu}^{\lambda}$ is a $T \times \mathbb{C}^{*}$-invariant open subset of the affine space $\mathbb{A}^{\frac{\lambda-\mu}{2}} \simeq \mathfrak{T}_{\mu}^{e} \cap \overline{\mathrm{Gr}}^{\lambda}$ containing the only $T \times \mathbb{C}^{*}$-fixed point $\mu$, and the action of $T \times \mathbb{C}^{*}$ on $\mathbb{A}^{\frac{\lambda-\mu}{2}}$ is linear with weights $\left\{-x-\frac{\lambda+\mu}{2} \hbar,-x+\hbar-\frac{\lambda+\mu}{2} \hbar, \ldots,-x-\hbar-\mu \hbar\right\}$ (see e.g. Section 5.2 of [3]). Similarly, $\mathfrak{T}_{\mu}^{s} \cap \overline{\mathcal{W}}_{\mu}^{\lambda}$ is a $T \times \mathbb{C}^{*}$-invariant open subset of the affine space $\mathbb{A}^{\frac{\lambda-\mu}{2}}$ with the linear $T \times \mathbb{C}^{*}$-action with weights $\left\{x-\hbar, x-2 \hbar, \ldots, x-\frac{\lambda-\mu}{2} \hbar\right\}$.

Comparing (2.3) and (2.4) with (2.2) we conclude that $\mathrm{A}_{e, s}^{S_{\mu}\left(V^{\lambda}\right)}(x)=A_{s, V^{\lambda}}(-x-\hbar)$.

2.3. Reduction to rank 1 . By construction of operators $\mathrm{A}$, for any $\mu \in \Lambda, V \in$ $\operatorname{Rep}(\check{G}), w_{1}, w_{2} \in W$ we have $\mathrm{A}_{w_{2}, w_{1} w_{2}}^{S_{\mu}(V)} \circ \mathrm{A}_{e, w_{2}}^{S_{\mu}(V)}=\mathrm{A}_{e, w_{1} w_{2}}^{S_{\mu}(V)}$, and also $\mathrm{A}_{w_{2}, w_{1} w_{2}}^{S_{\mu}(V)}(x)=$ $\mathrm{A}_{e, w_{1}}^{S_{\mu}(V)}\left(w_{2} x\right)$. In particular,

$$
\mathrm{A}_{e, w_{1}}^{S_{\mu}(V)}\left(w_{2} x\right) \circ \mathrm{A}_{e, w_{2}}^{S_{\mu}(V)}(x)=\mathrm{A}_{e, w_{1} w_{2}}^{S_{\mu}(V)}(x) .
$$

According to Lemma 17 of [5], in case length $l\left(w_{1} w_{2}\right)=l\left(w_{1}\right)+l\left(w_{2}\right)$, we have

$$
A_{w_{1}, V}\left(w_{2} x\right) \circ A_{w_{2}, V}(x)=A_{w_{1} w_{2}, V}(x) .
$$

Comparing (2.5) and (2.6) and choosing a reduced decomposition of $w \in W$ into a product of simple reflections, we see that it is enough to prove Theorem 1.6 for $w=s$ a simple reflection.

Let $G \supset P \rightarrow L$ be the corresponding (to $s$ ) submimimal parabolic subgroup containing $B_{-}$, and Levi group. We denote by $\breve{L} \subset \breve{G}$ the corresponding semisimple rank 1 Levi subgroup of $\check{G}$. The connected components of $\operatorname{Gr}_{P}$ are numbered by the lattice $\Lambda_{G, P}$ of characters of the center $Z(\check{L})$ of $\check{L}$. For $\theta \in \Lambda_{G, P}$ we denote by $\imath_{\theta}: \operatorname{Gr}_{P, \theta} \hookrightarrow \mathrm{Gr}_{G}$ the locally closed embedding of the corresponding connected component. We denote by $\mathfrak{p}^{\theta}: \mathrm{Gr}_{P, \theta} \rightarrow \mathrm{Gr}_{L, \theta}$ the projection to the corresponding connected component of the affine Grassmannian of $L$. Let $\check{\rho}_{G, L}$ stand for $\check{\rho}_{G}-\check{\rho}_{L}$. Then for a $G(\mathcal{O})$-equivariant perverse sheaf $\mathcal{F}$ on $\mathrm{Gr}_{G}$ the direct sum $\bigoplus_{\theta \in \Lambda_{G, P}} \mathfrak{p}_{*}^{\theta} l_{\theta} \mathfrak{F}\left[\left\langle\mu, 2 \check{\rho}_{G, L}\right\rangle\right]$ is a $L(\mathcal{O})$-equivariant perverse sheaf on $\mathrm{Gr}_{L}$ (see [2], Proposition 5.3.29) to be denoted by $R^{G, L} \mathcal{F}=\bigoplus_{\theta \in \Lambda_{G, P}} R_{\theta}^{G, L} \mathcal{F}$. Moreover, according to loc. cit., for any $V \in \operatorname{Rep}(\check{G})$ we have $R^{G, L} S(V) \cong S_{L}\left(\operatorname{Res}_{\check{L}}^{\check{G}} V\right)$ where $\operatorname{Res}_{\check{L}}^{\check{G}} V$ stands for the restriction of $V$ from $\check{G}$ to $\check{L}$.

Given $\mu \in \Lambda$ we will denote by $\bar{\mu}$ its image in the quotient lattice $\Lambda_{G, P}$. For a $T \times \mathbb{C}^{*}$-equivariant constructible complex on the slice $\mathcal{W}_{\mu}$, it is easy to see that $\mathrm{R}_{\bar{\mu}}^{G, L} \mathcal{F}=\mathfrak{p}_{*}^{\bar{\mu}} l_{\bar{\mu}} \mathcal{F}$ will be a $T \times \mathbb{C}^{*}$-equivariant constructible complex supported on $\mathcal{W}_{\mu}^{L}$ (the transversal slice on the affine Grassmannian of $L$ through the point $\mu$ ). For $V \in \operatorname{Rep}(\check{G}), \mu \in \Lambda^{+}$, and $w \in W_{L}=\{e, s\}$ by the base change we have $\mathrm{R}_{\mu}^{G, w} S_{\mu}^{G}(V)=\mathrm{R}_{\mu}^{w} S_{\mu}(V) \cong \mathrm{R}_{\mu}^{L, w} \mathrm{R}_{\bar{\mu}}^{G, L} S_{\mu}^{G}(V) \cong \mathrm{R}_{\mu}^{L, w} S_{\mu}^{L}\left(\operatorname{Res}_{\tilde{L}}^{\breve{G}} V\right)$ where we have added the superscripts ${ }^{G}$ or ${ }^{L}$ to $\mathrm{R}_{\mu}^{w}$ and $S_{\mu}$ in order to distinguish the hyperbolic stalks taken 
on transversal slices of the affine Grassmannians of $G$ of $L$. Moreover, $\mathrm{A}_{e, s}^{G, S_{\mu}^{G}(V)}=$ $\mathrm{A}_{e, s}^{L, S_{\mu}^{L}\left(\operatorname{Res}_{\tilde{L}}^{\check{G}} V\right)}$.

Now all the connected components of $\mathrm{Gr}_{L}$ are of the type considered in Section 2.2, and the $L(\mathcal{O})$-equivariant perverse sheaf $S_{\mu}^{L}\left(\operatorname{Res}_{\breve{L}}^{\breve{G}} V\right)$ is a direct sum of sheaves considered in Section 2.2. Finally, the action of the dynamical Weyl group operator $A_{s, V}$ is defined as $A_{s, \operatorname{Res}_{\tilde{L}}^{\check{G}} V}$. By the virtue of Section 2.2 we conclude that $\mathrm{A}_{e, s}^{S_{\mu}(V)}(x)=A_{s, V}(-x-\hbar \rho)$.

This completes the proof of Theorem 1.6.

\section{Affine case}

3.1. The group $G_{\text {aff }}$. From now on we assume that $G$ is almost simple and simply connected. To a connected reductive group $G$ as above one can associate the corresponding affine Kac-Moody group $G_{\text {aff }}$ in the following way. One can consider the polynomial loop group $G\left[t, t^{-1}\right]$ (this is an infinite-dimensional group ind-scheme)

It is well-known that $G\left[t, t^{-1}\right]$ possesses a canonical central extension $\widetilde{G}$ of $G\left[t, t^{-1}\right]$ :

$$
1 \rightarrow \mathbb{G}_{m} \rightarrow \widetilde{G} \rightarrow G\left[t, t^{-1}\right] \rightarrow 1
$$

Moreover, $\widetilde{G}$ has again a natural structure of a group ind-scheme.

The multiplicative group $\mathbb{G}_{m}$ acts naturally on $G\left[t, t^{-1}\right]$ and this action lifts to $\widetilde{G}$. We denote the corresponding semi-direct product by $G_{\text {aff }}$; we also let $\mathfrak{g}_{\text {aff }}$ denote its Lie algebra.

The Lie algebra $\mathfrak{g}_{\text {aff }}$ is an untwisted affine Kac-Moody Lie algebra. In particular, it can be described by the corresponding affine root system. We denote by $\mathfrak{g}_{\text {aff }}^{\vee}$ the $L a n g$ lands dual affine Lie algebra (which corresponds to the dual affine root system) and by $G_{\text {aff }}^{\vee}$ the corresponding dual affine Kac-Moody group, normalized by the property that it contains $G^{\vee}$ as a subgroup (cf. [4], Subsection 3.1 for more details).

We denote by $\Lambda_{\text {aff }}=\mathbb{Z} \times \Lambda \times \mathbb{Z}$ the coweight lattice of $G_{\text {aff }}$; this is the same as the weight lattice of $G_{\text {aff }}^{\vee}$. Here the first $\mathbb{Z}$-factor is responsible for the center of $G_{\text {aff }}^{\vee}$ (or $\widehat{G}^{\vee}$ ); it can also be thought of as coming from the loop rotation in $G_{\text {aff }}$. The second $\mathbb{Z}$-factor is responsible for the loop rotation in $G_{\text {aff }}^{\vee}$ it may also be thought of as coming from the center of $\left.G_{\text {aff }}\right)$. We denote by $\Lambda_{\text {aff }}^{+}$the set of dominant weights of $G_{\text {aff }}^{\vee}$ (which is the same as the set of dominant coweights of $G_{\text {aff }}$ ). We also denote by $\Lambda_{\text {aff }, k}$ the set of weights of $G_{\text {aff }}^{\vee}$ of level $k$, i.e. all the weights of the form $(k, \bar{\lambda}, n)$. We put $\Lambda_{\text {aff }, k}^{+}=\Lambda_{\text {aff }}^{+} \cap \Lambda_{\text {aff }, k}$.

Important notational convention: From now on we shall denote elements of $\Lambda$ by $\bar{\lambda}, \bar{\mu} \ldots$ (instead of just writing $\lambda, \mu \ldots$ in order to distinguish them from the coweights of $G_{\text {aff }}$ (= weights of $G_{\text {aff }}^{\vee}$ ), which we shall just denote by $\lambda, \mu \ldots$

Let $\Lambda_{k}^{+} \subset \Lambda$ denote the set of dominant coweights of $G$ such that $\langle\bar{\lambda}, \alpha) \leq k$ when $\alpha$ is the highest root of $\mathfrak{g}$. Then it is well-known that a weight $(k, \bar{\lambda}, n)$ of $G_{\text {aff }}^{\vee}$ lies in $\Lambda_{\text {aff }, k}^{+}$if and only if $\bar{\lambda} \in \Lambda_{k}^{+}$(thus $\Lambda_{\text {aff }, k}=\Lambda_{k}^{+} \times \mathbb{Z}$ ).

Let also $W_{\text {aff }}$ denote affine Weyl group of $G$ which is the semi-direct product of $W$ and $\Lambda$. It acts on the lattice $\Lambda_{\text {aff }}($ resp. $\widehat{\Lambda})$ preserving each $\Lambda_{\text {aff, } k}\left(\right.$ resp. each $\left.\widehat{\Lambda}_{k}\right)$. In order to describe this action explicitly it is convenient to set $W_{\text {aff }, k}=W \ltimes k \Lambda$ which naturally acts on $\Lambda$. Of course the groups $W_{\text {aff }, k}$ are canonically isomorphic to $W_{\text {aff }}$ 


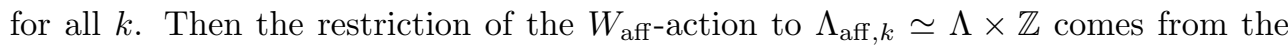

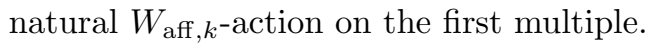

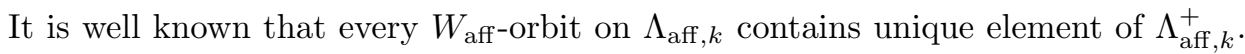
This is equivalent to saying that $\Lambda_{k}^{+} \simeq \Lambda / W_{\text {aff }, k}$.

3.2. Repellents. Our dream is to create an analog of the affine Grassmannian $\mathrm{Gr}_{G}$ and the above results about it in the case when $G$ is replaced by the (infinitedimensional) group $G_{\text {aff }}$. The first attempt to do so was made in [4]: namely, in loc. cit. we have constructed analogs $\overline{\mathcal{W}}_{\mu}^{\lambda}$ of the varieties $\overline{\mathcal{W}} \bar{\mu}$ in the case when $G$ is replaced by $G_{\text {aff }}$. We will not reproduce the (rather involved) definition of $\overline{\mathcal{W}}_{\mu}^{\lambda}$ here; we only mention that the open pieces $\mathcal{W}_{\mu}^{\lambda}$ are the moduli spaces of certain $G$-bundles on $\mathbb{P}^{2}$ trivialized at infinity $\mathbb{P}^{1} \subset \mathbb{P}^{2}$. The complement $\mathbb{A}^{2}=\mathbb{P}^{2} \backslash \mathbb{P}^{1}$ is equipped with coordinates $(z, t)$, and with the action of $\mathbb{C}^{*} \times \mathbb{C}^{*}:(a, b) \cdot(z, t)=(a z, b t)$. The 2-dimensional torus $\mathbb{C}^{*} \times \mathbb{C}^{*}$ acts on $\overline{\mathcal{W}}_{\mu}^{\lambda}$ by transport of structure, and $T \subset G$ acts via trivialization at infinity. Let $\mathbb{C}_{\text {hyp }}^{*}:=\left\{\left(c, c^{-1}\right)\right\} \subset \mathbb{C}^{*} \times \mathbb{C}^{*}\left(\right.$ resp. $\left.\mathbb{C}_{\Delta}^{*}:=\{(c, c)\} \subset \mathbb{C}^{*} \times \mathbb{C}^{*}\right)$ stand for the antidiagonal, alias hyperbolic, (resp. diagonal) subgroup. Let us denote the torus $\mathbb{C}_{\text {hyp }}^{*} \times T$ by $\widehat{T}$. Thus, the slices $\overline{\mathcal{W}}_{\mu}^{\lambda}$ are equipped with the action of the torus $\widehat{T} \times \mathbb{C}_{\Delta}^{*}$. The Lie algebra of $\widehat{T}$ is denoted by $\widehat{\mathfrak{t}}$.

The cocharacter lattice $X_{*}(\widehat{T}) \cong \mathbb{Z} \times \Lambda \subset \mathbb{Z} \times \Lambda \times \mathbb{Z}=X_{*}\left(T_{\text {aff }}\right)$. Accordingly, we have $\widehat{T} \subset T_{\text {aff }}$. We propose that the action of $\widehat{T}$ on $\overline{\mathcal{W}}_{\mu}^{\lambda}$ of the previous paragraph is nothing else than the action coming from the $T_{\text {aff-action on the transversal slices }}$ of the double affine Grassmannian. Also, the action of $\mathbb{C}_{\Delta}^{*}$ on $\overline{\mathcal{W}}_{\mu}^{\lambda}$ of the previous paragraph is nothing but the loop rotation action on the transversal slices of the double affine Grassmannian.

Let $I$ (resp. $I_{\text {aff }}=I \sqcup i_{0}$ ) stand for the set of vertices of the Dynkin diagram of $G$ (resp. of $G_{\text {aff }}$ ). For $i \in I$ we denote by $\bar{\omega}_{i} \in X_{*}(T)$ the corresponding fundamental coweight of $G$, and we denote by $a_{i} \in \mathbb{N}$ the corresponding label of the Dynkin diagram of $G_{\text {aff }}$. Then $\omega_{i_{0}}:=(1,0) \in \mathbb{Z} \times X_{*}(T)=X_{*}(\widehat{T}), \omega_{i}:=\left(a_{i}, \bar{\omega}_{i}\right) \in X_{*}(\widehat{T}), i \in I$, are the fundamental coweights of $G_{\text {aff }}$. We set $\rho:=\sum_{i \in I_{\text {aff }}} \omega_{i}$ (not to be confused with the halfsum $\bar{\rho}$ of positive coroots of $G$ ). The group $W_{\text {aff }, k}$ acts on $X_{*}(\widehat{T})$, and for $w \in W_{\text {aff }, k}$ let us view $w \rho$ as a one-parametric subgroup $\mathbb{C}^{*} \rightarrow \widehat{T}$. The torus $\widehat{T}$ acts on $\overline{\mathcal{W}}_{\mu}^{\lambda}$ with the only fixed point, to be denoted abusively by $\mu$, and by analogy with Section 2.1, we define $\mathfrak{T}_{\mu}^{w} \subset \overline{\mathcal{W}}_{\mu}^{\lambda}$ as the repellent $\mathfrak{T}_{\mu}^{w}:=\left\{g \in \overline{\mathcal{W}}_{\mu}^{\lambda}: \lim _{c \rightarrow \infty} w \rho(c) g=\mu\right\}$. For $G=S L(N)$ these repellents were considered by H. Nakajima in Section 6 of [11] under the name of MV cycles.

The same procedure as in Section 1.3 defines an isomorphism $\mathrm{A}_{w, y}^{\mathrm{IC}\left(\overline{\mathcal{W}}_{\mu}^{\lambda}\right)}: \quad \mathrm{R}_{\mu}^{w} \mathrm{IC}\left(\overline{\mathcal{W}}_{\mu}^{\lambda}\right) \otimes_{\mathbb{C}\left[\hat{\mathfrak{t}} \times \mathbb{A}^{1}\right]} \mathbb{C}\left(\widehat{\mathfrak{t}} \times \mathbb{A}^{1}\right) \stackrel{\sim}{\longrightarrow} \mathrm{R}_{\mu}^{y} \operatorname{IC}\left(\overline{\mathcal{W}}_{\mu}^{\lambda}\right) \otimes_{\mathbb{C}\left[\widehat{\mathfrak{t}} \times \mathbb{A}^{1}\right]} \mathbb{C}\left(\widehat{\mathfrak{t}} \times \mathbb{A}^{1}\right)$. Here $\mathbb{A}^{1}$ is the affine line with coordinate $\hbar$, the positive generator of the integral equivariant cohomology $H_{\mathbb{C}_{\Delta}^{*}}^{2}(p t, \mathbb{Z})$.

Conjecture 3.3. (a) For $\lambda, \mu \in \Lambda_{k}^{+}, w \in W_{\mathrm{aff}, k}$, we have $\mathrm{R}_{\mu}^{w} \operatorname{IC}\left(\overline{\mathcal{W}}_{\mu}^{\lambda}\right) \cong V_{w \mu}^{\lambda} \otimes \mathbb{C}\left[\hat{\mathfrak{t}} \times \mathbb{A}^{1}\right]$ where $V_{\mu}^{\lambda}$ stands for the $w \mu$-weight space of the irreducible integrable $G_{\mathrm{aff}}^{\vee}$-module with highest weight $\lambda$; 
(b) We have $\mathrm{A}_{e, w}^{\mathrm{IC}\left(\overline{\mathcal{W}}_{\mu}^{\lambda}\right)}(x)=A_{w, V^{\lambda}}(-x-\hbar \rho)$ where $A_{w, V^{\lambda}}$ stands for the dynamical affine Weyl group action of [5].

As was mentioned in the Introduction, in the case of representations of level 1, one can probably deduce Conjecture 3.3 for $G=S L(n)$ (as well as some generalization of Conjecture 3.3 to $G=G L(n)$ ) from [9] (by using also the results of [4] and [11]).

\section{References}

[1] R. Asherova, Yu. Smirnov, V. Tolstoy, Projection operators for simple Lie groups, Theor. Math. Phys. 8 (1971), 813-825.

[2] A. Beilinson and V. Drinfeld, Quantization of Hitchin's Hamiltonians and Hecke eigen-sheaves, Preprint, available at http://www.math.uchicago.edu/ $\sim$ mitya/langlands.html

[3] R. Bezrukavnikov, M. Finkelberg, Equivariant Satake Category and Kostant-Whittaker Reduction, Moscow Math. Jour. 8 (2008), 39-72.

[4] A. Braverman and M. Finkelberg, Pursuing the double affine Grassmannian I: Transversal slices via instantons on $A_{k}$-singularities, Duke Math. J. 152 (2010), 175-206.

[5] P. Etingof, A. Varchenko, Dynamical Weyl groups and Applications, Adv. Math. 167 (2002), $74-127$.

[6] V. Ginzburg, Perverse sheaves on a loop group and Langlands duality, Preprint, alggeom/9511007.

[7] M. Kashiwara, T. Tanisaki, Kazhdan-Lusztig conjecture for affine Lie algebras with negative level, Duke Math. J. 77 (1995), 21-62.

[8] G. Lusztig, Singularities, character formulas, and a q-analog of weight multiplicities, Analysis and topology on singular spaces, Astérisque, 101-102 (1983), 208-229.

[9] D. Maulik and A. Okounkov, in preparation.

[10] I. Mirković and K. Vilonen, Geometric Langlands duality and representations of algebraic groups over commutative rings, Annals of Math. (2) 166 (2007), 95-143.

[11] H. Nakajima, Quiver varieties and branching, SIGMA 5 (2009), 003, 37 pages.

[12] D. P. Zhelobenko, Extremal projectors and generalized Mickelsson algebras on reductive Lie algebras, Math. USSR, Izv. 33 (1989), 85-100.

Department of Mathematics, Brown University, 151 Thayer St., Providence Ri 02912, USA

E-mail address: braval@math.brown.edu

imu, iITP and State University Higher School of Economics, Department of MatheMatics, 20 Myasnitskaya St, Moscow 101000 Russia

E-mail address: fnklberg@gmail.com 